\title{
Experimental Implementation of Real-Time Non-Orthogonal Multi-Carrier Systems in a Realistic Fading Channel
}

\author{
Waseem Ozan, Hedaia Ghannam, Paul Anthony Haigh and Izzat Darwazeh \\ Department of Electronic and Electrical Engineering, \\ University College London, London, UK \\ \{w.ozan; hedaia.ghannam.15; p.haigh; i.darwazeh\}@ucl.ac.uk
}

\begin{abstract}
Spectrally efficient frequency division multiplexing (SEFDM) has the potential to improve spectrum utilisation through bandwidth compression at the cost of self-induced intercarrier interference. In this paper, an experimental test-bed is designed and implemented to evaluate the performance of SEFDM systems in real-time using frequency-domain channel estimation/equalisation and iterative signal detection. Our innovation lies in the development and experimental, real-time implementation of baseband generation, signal assembly, signal decoding and a novel frequency-domain channel estimation and equalisation method for the SEFDM transceiver. Our system compresses the transmitted signal bandwidth up to $60 \%, 30 \%$ and $20 \%$ for BPSK, QPSK and 8PSK respectively, offering significant bandwidth savings, and moving towards satisfying one of the key $5 \mathrm{G}$ challenges.
\end{abstract}

\section{INTRODUCTION}

In future $(5 \mathrm{G})$ networks, user demand is expected to be significantly in excess of the total current network supply. Although future 5G standards are still not fixed, one of the most important requirements is higher spectrum efficiency. To achieve this, either the frequency distance between subcarriers is scaled down to save bandwidth, breaking the orthogonality limit, or the data is sent faster. Generally, both techniques result in non-orthogonal signals, that could either save bandwidth or time.

During the last decade, spectrally efficient frequency division multiplexing (SEFDM) signals and systems have been proposed to reduce the spacing between the subcarriers and pack more subcarriers into the available bandwidth [1], whilst avoiding the degradation of the bit error rate (BER), relative to orthogonal frequency division multiplexing (OFDM). Similarly, multi-stream faster-than-Nyquist (MFTN) systems have been suggested to improve the spectral efficiency beyond the Nyquist limit [2] [3]. Recently, a new waveform termed truncated OFDM (TOFDM) is proposed [4], where the transmission rate is increased without sacrificing bandwidth by partial time transmission of OFDM symbols, resulting in a shorter transmission time for OFDM symbols and frames to improve the data rate.

One of the major disadvantages of SEFDM is its high computationally complex receivers. The two main suggestions to reduce computational complexity are, $(i)$ employing channel estimation and equalisation in the frequency-domain, and (ii) applying a low complexity coded iterative detector [5] [6], which employs convolutional coding and the turbo principle.

This paper is organised as follows, Section II describes the experimental setup for the SEFDM system under test. The measured BER results are used to evaluate the system performance and are shown in Section III. Finally, Section IV concludes the paper.

\section{EXPERIMENTAL SETUP}

This section presents the software and hardware setup of the real time experiment for evaluation of SEFDM systems in the Long Term Evolution (LTE) EPA5 channel. In general, the test-bed of this experiment consists of universal software radio peripheral (USRP) transceivers (NI USRP RIO N2395R) programmed using LabVIEW, plus, a Spirent VR5 channel emulator to generate realistic LTE channels. Both software and hardware specifications are detailed below.

\section{A. Design and Setup System Transmitter}

At the transmitter, a stream of pseudorandom bits $U 1$ is generated before coding. This experiment employs a recursive systematic convolutional coder with code rate $R_{c}=1 / 2$, followed by a block interleaver before being mapped onto the appropriate constellation (shown in blue in Fig. 2). In this work, we test binary phase shift keying (BPSK), quadrature phase shift keying (QPSK) and 8-phase shift keying (8-PSK).

Next, the complex data is divided into parallel streams by a serial-to-parallel converter. The parallel streams are fed to

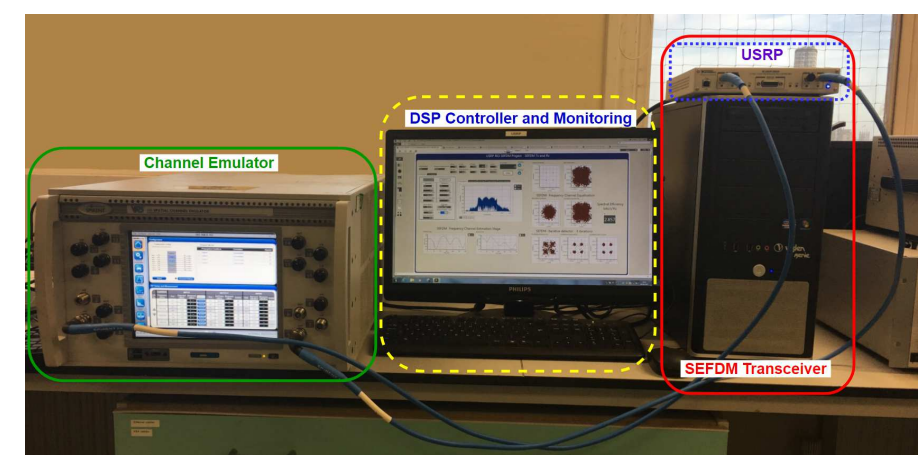

Fig. 1. SEFDM transceiver test-bed setup 


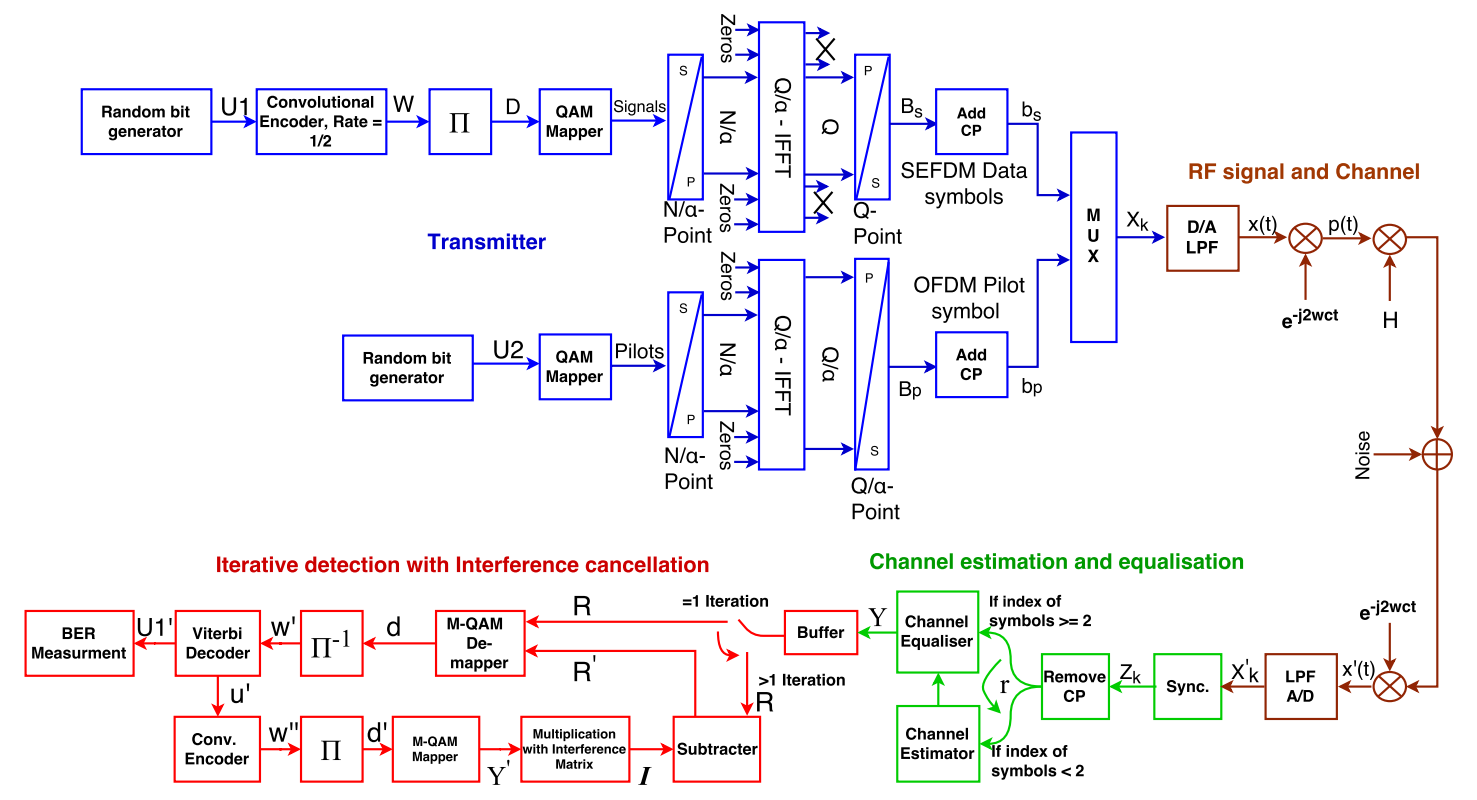

Fig. 2. Schematic block diagram of SEFDM system

an inverse fast Fourier transform (IFFT) of length (the upper one in the blue part in Fig. 2). After that, a cyclic prefix (CP) is added at the beginning of every transmitted symbol. In this work, the symbols are transmitted in frames, where each frame consists of a pilot symbol followed by 24 data symbols.

In the final stage of the transmitter, the complex baseband signal, $X_{k}$, is fed to an FPGA contained within the aforementioned USRP to perform digital-to-analogue (D/A) conversion and up-conversion to the $2 \mathrm{GHz}$ band. Table I depicts the system parameters of this experiment.

TABLE I

EXPERIMENTAL SYSTEM SPECIFICATIONS

\begin{tabular}{ll}
\hline Parameters & Values \\
\hline \hline Central carrier frequency & $2 \mathrm{GHz}$ \\
\hline Sampling frequency & $30.72 \mathrm{MHz}$ \\
\hline Signal bandwidth & $18 \mathrm{MHz}$ \\
\hline Values of $\alpha$ & $1(\mathrm{OFDM}) ; 0.9 ; \ldots ; 0.4$ \\
\hline Subcarrier baseband bandwidth & $60 \mathrm{KHz}$ \\
\hline Subcarrier spacing & $\alpha \times 60 \mathrm{KHz}$ \\
\hline IFFT/FFT size & 512 \\
\hline Cyclic prefix & 128 time samples \\
\hline Modulation scheme & BPSK; QPSK; 8-PSK \\
\hline
\end{tabular}

\section{B. LTE Fading Channel Model and Channel Estimation and Equalisation}

The RF signal is transmitted through a VR5 channel emulator that has LTE EPA5 wireless channel model [9]. The signal output of the VR5 is fed to the USRP receiver to deliver the baseband digital domain signal. A Schmidl and Cox [10] synchronization is applied in this experiment, where two identical timing sequences are added at the start of each frame to estimate the first sample of the data symbols.

Following this, the $\mathrm{CP}$ is removed from the received symbols and the first symbol of $r$, the OFDM pilot symbol is fed to a single tap channel estimator. Consequently, the channel estimator output with the data symbols are fed to the channel equaliser to mitigate the phase and amplitude distortion from the signal.

\section{Iterative Detector with Interference Canceller}

At the detection stage, an iterative detector with an interference canceller are utilised to retrieve the data. The equalised signal, $Y$ is de-mapped and de-interleaved before viterbi decoding. Next, the recovered, decoded data, in combination with the knowledge of the correlation matrix, are used to estimate the interference, $I$ generated in SEFDM due to the compression of the subcarriers spacing. The correlation matrix contains information about the contribution of the interference from all the subcarriers to to each subcarrier [11]. After subtracting the estimated interference, the signal, $R^{\prime}$ is sent to the next iteration to get an enhanced version of the estimated signal; repetition of this process leads to a better signal estimates. A buffer stores the equalised symbols, $Y$ until a specified number of iterations have been performed.

\section{EXPERIMENTAL RESULTS}

The measured BER of BPSK, QPSK and 8PSK-SEFDM are shown in Fig. 3, Fig. 4 and Fig. 5, respectively.

It is evident that SEFDM systems are compatible with varying degrees of modulation cardinality. For instance, in Fig. 3, a BPSK-SEFDM system can achieve sufficiently low BERs at $\alpha \longrightarrow 0.4$, where a power penalty of approximately $2 \mathrm{~dB}$ is observed. In terms of trade-off for the improved spectral efficiency obtained using this particular system, such a power penalty may be tolerable.

From Fig. 4, it should be noted that at a compression factor of $20 \%$, i.e. $\alpha=0.8$, QPSK-SEFDM shows pseudo-equivalent 
performance in comparison to conventional OFDM, where less than $1 \mathrm{~dB}$ power penalty is observed. Furthermore, the 8-PSKSEFDM system works for $\alpha=0.8$, with a power penalty $<3 \mathrm{~dB}$. The results from Fig. 4 and Fig. 5 also show that subcarriers can be compressed to a limit where an error floor appears at low values of $\alpha$, such as $\alpha \sim 0.6$ for QPSK-SEFDM and $\alpha \sim 0.7$ for 8PSK-SEFDM.

\section{CONCLUSION}

In SEFDM systems, which demonstrate significant spectral efficiency gains, real-time system implementation is a challenge due to the combined impact of inter-carrier interference and multi-path effects. The paper introduces a world-first, experimental real-time implementation of baseband generation, signal assembly, signal decoding and a novel frequencydomain channel estimation and equalisation method, where OFDM pilot symbol precedes the SEFDM information signal to facilitate accurate frequency-domain channel estimation. Such experimental results were obtained by testing signals over the LTE EPA5 fading channel generated by a VR5channel emulator operating at a frequency of $2 \mathrm{GHz}$.

\section{ACKNOWLEDGMENT}

This work is part funded by UCL's EPSRC "Impact Acceleration Discovery to Use" Award for the development of pre-commercialization $5 \mathrm{G}$ transceiver prototype and by National Instruments and a generous donation of LTE FPGA core through Xilinx University Donation program. Dr Paul Anthony Haigh is supported by the UK EPSRC through grant EP/P006280/1 (MARVEL), Waseem Ozan and Hedaia Ghannam's PhD studies are supported by UCL.

\section{REFERENCES}

[1] I. Kanaras, A. Chorti, M. R. D. Rodrigues and I. Darwazeh, "Spectrally Efficient FDM Signals: Bandwidth Gain at the Expense of Receiver Complexity,“. Communications, 2009. ICC '09. IEEE International Conference on, Dresden, 2009, pp. 1-6.

[2] D. Dasalukunte, F. Rusek and V. Owall, "Multicarrier Faster-ThanNyquist Transceivers: Hardware Architecture and Performance Analysis," in IEEE Transactions on Circuits and Systems I: Regular Papers, vol. 58, no. 4, pp. 827-838, April 2011.

[3] J. B. Anderson, F. Rusek and V. wall, "Faster-Than-Nyquist Signaling," in Proceedings of the IEEE, vol. 101, no. 8, pp. 1817-1830, Aug. 2013.

[4] W. Ozan, K. Jamieson and I. Darwazeh, "Truncating and oversampling OFDM signals in white Gaussian noise channels," 2016 10th International Symposium on Communication Systems, Networks and Digital Signal Processing (CSNDSP), Prague, 2016, pp. 1-6.

[5] T. Xu and I. Darwazeh, "A Soft Detector for Spectrally Efficient Systems With Non-Orthogonal Overlapped Sub-Carriers," in IEEE Communications Letters, vol. 18, no. 10, pp. 1847-1850, Oct. 2014.

[6] J. Hagenauer, The turbo principle: Tutorial introduction and state of the art, in Proc. Int. Symp. Turbo Codes, Sep. 1997, pp. 111.

[7] S. Isam and I. Darwazeh, "Robust channel estimation for Spectrally Efficient FDM system," 2012 19th International Conference on Telecommunications (ICT), Jounieh, 2012, pp. 1-6.

[8] T. Xu and I. Darwazeh, "Transmission Experiment of Bandwidth Compressed Carrier Aggregation in a Realistic Fading Channel," in IEEE Transactions on Vehicular Technology, vol. 66, no. 5, pp. 4087-4097, May 2017.

[9] Evolved Universal Terrestrial Radio Access (E-UTRA); Base station (BS) radio transmission and reception, 3GPP TS 36.104 V10.2.0, May 2011.

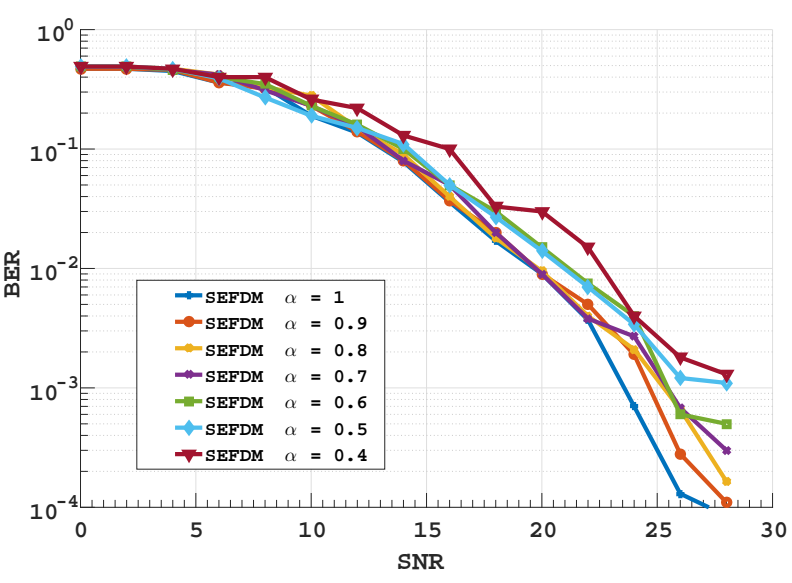

Fig. 3. BER of BPSK-SEFDM using OFDM pilots

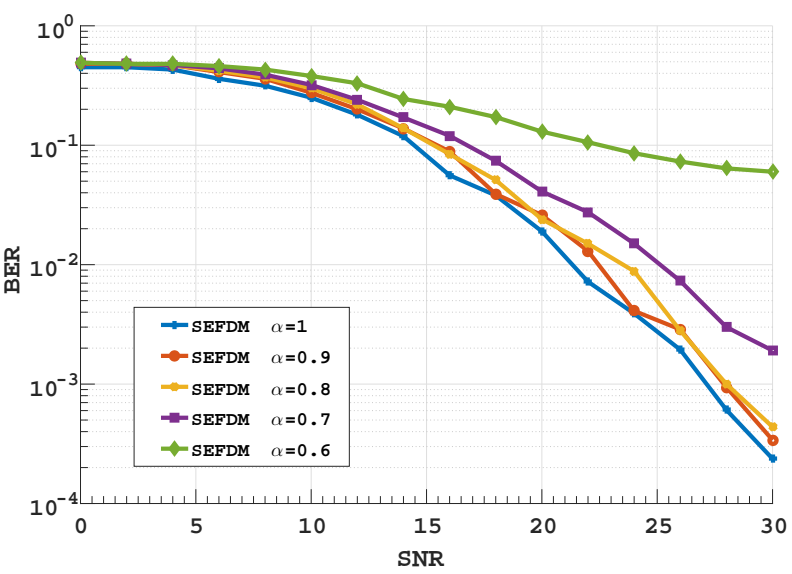

Fig. 4. BER of QPSK-SEFDM using OFDM pilots

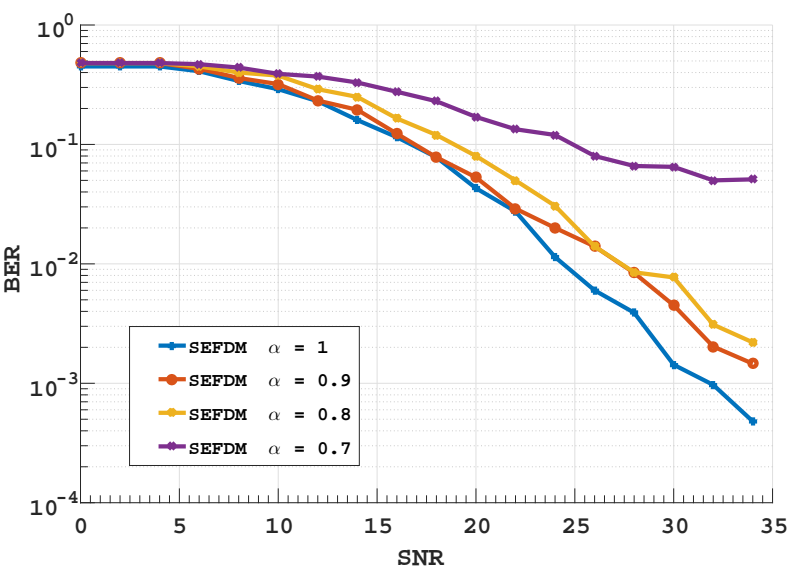

Fig. 5. BER of 8-PSK-SEFDM using OFDM pilots

[10] T. M. Schmidl and D. C. Cox, "Robust frequency and timing synchronization for OFDM," in IEEE Transactions on Communications, vol. 45 no. 12, pp. 1613-1621, Dec 1997.

[11] S. Isam and I. Darwazeh, "Characterizing the intercarrier interference of non-orthogonal Spectrally Efficient FDM system," 2012 8th International Symposium on Communication Systems, Networks \& Digital Signal Processing (CSNDSP), Poznan, 2012, pp. 1-5. 